VIII JORNADAS

SOBRE INNOVACIÓN DOCENTE

EN ARQUITECTURA

WORKSHOP ON EDUCATIONAL INNOVATION

IN ARCHITECTURE JIDA'20

JORNADES SOBRE INNOVACIÓ

DOCENT EN ARQUITECTURA JIDA'20

ESCUELA TÉCNICA SUPERIOR DE ARQUITECTURA DE MÁLAGA 12 Y 13 DE NOVIEMBRE DE 2020 


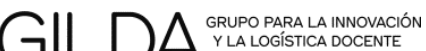 EN ARQUITECTURA}

Organiza e impulsa GILDA (Grupo para la Innovación y Logística Docente en la Arquitectura), en el marco del proyecto RIMA (Investigación e Innovación en Metodologías de Aprendizaje), de la Universitat Politècnica de Catalunya - BarcelonaTech (UPC) y el Institut de Ciències de l'Educació (ICE). http://revistes.upc.edu/ojs/index.php/JIDA

\section{Editores}

Berta Bardí i Milà, Daniel García-Escudero

\section{Revisión de textos}

Alba Arboix, Jordi Franquesa, Joan Moreno, Judit Taberna

\section{Edita}

Iniciativa Digital Politècnica Oficina de Publicacions Acadèmiques Digitals de la UPC Publicaciones y Divulgación Científica, Universidad de Málaga

ISBN 978-84-9880-858-2 (IDP-UPC) 978-84-1335-032-5 (UMA EDITORIAL)

eISSN 2462-571X

(C) de los textos y las imágenes: los autores

(C) de la presente edición: Iniciativa Digital Politècnica Oficina de Publicacions Acadèmiques Digitals de la UPC, UMA

Esta obra está sujeta a una licencia Creative Commons:

Reconocimiento - No comercial - SinObraDerivada (cc-by-nc-nd):

http://creativecommons.org/licences/by-nc-nd/3.0/es

https://creativecommons.org/licenses/by-nc-nd/4.0/

Cualquier parte de esta obra se puede reproducir sin autorización pero con el reconocimiento y atribución de los autores.

No se puede hacer uso comercial de la obra y no se puede alterar, transformar o hacer obras derivadas. 


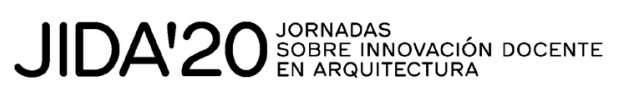

\section{Comité Organizador JIDA'20}

\section{Dirección y edición}

\section{Berta Bardí i Milà (GILDA)}

Dra. Arquitecta, Departamento de Proyectos Arquitectónicos, ETSAB-UPC

Daniel García-Escudero (GILDA)

Dr. Arquitecto, Departamento de Proyectos Arquitectónicos, ETSAB-UPC

\section{Organización}

\section{Antonio Álvarez Gil}

Dr. Arquitecto, Departamento Arte y Arquitectura, eAM'-UMA

\section{Jordi Franquesa (Coordinador GILDA)}

Dr. Arquitecto, Departamento de Urbanismo y Ordenación del Territorio, ETSAB-UPC Joan Moreno Sanz (GILDA)

Dr. Arquitecto, Departamento de Urbanismo y Ordenación del Territorio, ETSAV-UPC

\section{Fernando Pérez del Pulgar Mancebo}

Dr. Arquitecto, Departamento Arte y Arquitectura, eAM'-UMA

Judit Taberna (GILDA)

Arquitecta, Departamento de Representación Arquitectónica, ETSAB-UPC

Ferran Ventura Blanch

Dr. Arquitecto, Departamento Arte y Arquitectura, eAM'-UMA

\section{Coordinación}

\section{Alba Arboix}

Dra. Arquitecta, Teoría e Historia de la Arquitectura y Técnicas de la Comunicación, ETSAB-UPC

\section{Comunicación}

Eduard Llorens i Pomés

ETSAB-UPC 


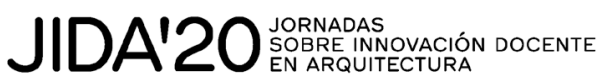

\section{Comité Científico JIDA'20}

Luisa Alarcón González

Dra. Arquitecta, Proyectos Arquitectónicos, ETSA-US

Gaizka Altuna Charterina

Arquitecto, Representación Arquitectónica y Diseño, TU Berlin

Atxu Amann Alcocer

Dra. Arquitecta, Ideación Gráfica Arquitectónica, ETSAM-UPM

Irma Arribas Pérez

Dra. Arquitecta, Diseño, Instituto Europeo de Diseño, IED Barcelona

Raimundo Bambó

Dr. Arquitecto, Urbanismo y ordenación del territorio, EINA-UNIZAR

Iñaki Bergera

Dr. Arquitecto, Proyectos Arquitectónicos, EINA-UNIZAR

Jaume Blancafort

Dr. Arquitecto, Arquitectura y Tecnología de la Edificación, ETSAE-UPCT

Enrique Manuel Blanco Lorenzo

Dr. Arquitecto, Proyectos Arquitectónicos, Urbanismo y Composición, ETSAC-UdC

Francisco Javier Boned Purkiss

Dr. Arquitecto, Composición arquitectónica, eAM'-UMA

Ivan Cabrera i Fausto

Dr. Arquitecto, Mecánica de los Medios Continuos y Teoría de Estructuras, ETSA-UPV

Raúl Castellanos Gómez

Dr. Arquitecto, Proyectos Arquitectónicos, ETSA-UPV

Nuria Castilla Cabanes

Dra. Arquitecta, Construcciones arquitectónicas, ETSA-UPV

David Caralt

Arquitecto, Universidad San Sebastián, Sede Concepción, Chile

Rodrigo Carbajal Ballell

Dr. Arquitecto, Proyectos Arquitectónicos, ETSA-US

Eva Crespo

Dra. Arquitecta, Tecnología de la Arquitectura, ETSAB-UPC

Valentina Cristini

Dra. Arquitecta, Composición Arquitectónica, Instituto de Restauración del Patrimonio, ETSA-UPV

Silvia Colmenares

Dra. Arquitecta, Proyectos Arquitectónicos, ETSAM-UPM

Còssima Cornadó Bardón

Dra. Arquitecta, Tecnología de la Arquitectura, ETSAB-UPC

Eduardo Delgado Orusco

Dr. Arquitecto, Proyectos Arquitectónicos, EINA-UNIZAR

Carmen Díez Medina

Dra. Arquitecta, Composición, EINA-UNIZAR

Débora Domingo Calabuig

Dra. Arquitecta, Proyectos Arquitectónicos, ETSA-UPV 


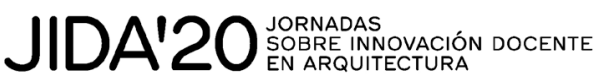

Maria Pia Fontana

Dra. Arquitecta, Arquitectura e Ingeniería de la Construcción, EPS-UdG

Arturo Frediani Sarfati

Dr. Arquitecto, Proyectos, Urbanismo y Dibujo, EAR-URV

Jessica Fuentealba Quilodrán

Arquitecta, Departamento Diseño y Teoría de la Arquitectura, Universidad del Bio-Bío, Concepción, Chile

Pedro García Martínez

Dr. Arquitecto, Arquitectura y Tecnología de la Edificación, ETSAE-UPCT

Mariona Genís Vinyals

Dra. Arquitecta, BAU Centre Universitari de Disseny, UVic-UCC

Eva Gil Lopesino

Arquitecta, Proyectos Arquitectónicos, ETSAM-UPM

María González

Arquitecta, Proyectos Arquitectónicos, ETSA-US

Arianna Guardiola Villora

Dra. Arquitecta, Mecánica de los Medios Continuos y Teoría de Estructuras, ETSA-UPV

Íñigo Lizundia Uranga

Dr. Arquitecto, Construcciones Arquitectónicas, ETSA EHU-UPV

Emma López Bahut

Dra. Arquitecta, Proyectos, Urbanismo y Composición, ETSAC-UdC

Juanjo López de la Cruz

Arquitecto, Proyectos Arquitectónicos, ETSA-US

Luis Machuca Casares

Dr. Arquitecto, Expresión Gráfica Arquitectónica, eAM'-UMA

Magda Mària Serrano

Dra. Arquitecta, Proyectos Arquitectónicos, ETSAV-UPC

Cristina Marieta Gorriti

Dra. Arquitecta, Ingeniería Química y del Medio Ambiente, EIG UPV-EHU

Marta Masdéu Bernat

Dra. Arquitecta, Arquitectura e Ingeniería de la Construcción, EPS-UdG

Camilla Mileto

Dra. Arquitecta, Composición arquitectónica, ETSA-UPV

Zaida Muxí Martínez

Dra. Arquitecta, Urbanismo y ordenación del territorio, ETSAB-UPC

David Navarro Moreno

Dr. Arquitecto, Arquitectura y Tecnología de la Edificación, ETSAE-UPCT

Luz Paz Agras

Dra. Arquitecta, Proyectos, Urbanismo y Composición, ETSAC-UdC

Oriol Pons Valladares

Dr. Arquitecto, Tecnología a la Arquitectura, ETSAB-UPC

Jorge Ramos Jular

Dr. Arquitecto, Teoría de la Arquitectura y Proyectos Arquitectónicos, ETSAVA-UVA

Amadeo Ramos Carranza

Dr. Arquitecto, Proyectos Arquitectónicos, ETSA-US 


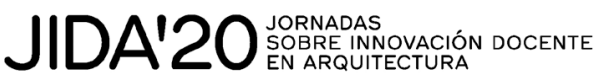

\section{Patricia Reus}

Dra. Arquitecta, Arquitectura y Tecnología de la Edificación, ETSAE-UPCT

\section{Antonio S. Río Vázquez}

Dr. Arquitecto, Composición arquitectónica, ETSAC-UdC

\section{Silvana Rodrigues de Oliveira}

Arquitecta, Proyectos Arquitectónicos, ETSA-US

Carlos Jesús Rosa Jiménez

Dr. Arquitecto, Urbanismo y ordenación del territorio, eAM'-UMA

\section{Jaume Roset Calzada}

Dr. Físico, Física Aplicada, ETSAB-UPC

\section{Patricia Sabín Díaz}

Dra. Arquitecta, Proyectos Arquitectónicos, Urbanismo y Composición, ETSAC-UdC

\section{Mara Sánchez Llorens}

Dra. Arquitecta, Ideación Gráfica Arquitectónica, ETSAM-UPM

\section{Carla Sentieri Omarrementeria}

Dra. Arquitecta, Proyectos Arquitectónicos, ETSA-UPV

\section{Marta Serra Permanyer}

Dra. Arquitecta, Teoría e Historia de la Arquitectura y Técnicas de la Comunicación, ETSAV-UPC

Sergio Vega Sánchez

Dr. Arquitecto, Construcción y Tecnologías Arquitectónicas, ETSAM-UPM

José Vela Castillo

Dr. Arquitecto, Culture and Theory in Architecture and Idea and Form, IE School of Architecture and Design, IE University, Segovia

\section{Isabel Zaragoza de Pedro}

Dra. Arquitecta, Representación Arquitectónica, ETSAB-UPC 


\section{ÍNDICE}

1. Coronawar. La docencia como espacio de resistencia. Coronawar. Teaching as a space of resistance. Ruiz-Plaza, Angela; De Coca-Leicher, José; Torrego-Gómez, Daniel.

2. Narrativa gráfica: el aprendizaje comunicativo del dibujar. Graphic narrative: the communicative learning of drawing. Salgado de la Rosa, María Asunción; Raposo Grau, Javier Fcob; Butragueño Díaz-Guerra, Belén.

3. Sobre la casa desde casa: nueva experiencia docente en la asignatura Taller de Arquitectura. About the house from home: new teaching experience in the subject Architecture Workshop. Millán-Millán, Pablo Manuel.

4. Muéstrame Málaga: Un recorrido por la historia de la arquitectura guiado por el alumnado. Show me Malaga: A tour through the history of architecture guided by students. González-Vera, Víctor Miguel.

5. Formaciones Feedback. Tres proyectos con materiales granulares manipulados robóticamente. Feedback Formation. Three teaching projects on robotically manipulated granular materials. Medina-lbáñez, Jesús; Jenny, David; Gramazio, Fabio; Kohler, Matthias.

6. La novia del Maule, recreación a escala 1:1. The Maule's Bride, recreation 1:1 scale. Zúñiga-Alegría, Blanca.

7. Docencia presencial con evaluación virtual. La adaptación del sistema de evaluación. On-site teaching with on-line testing. The adaptation of the evaluation system. Navarro-Moreno, David; La Spina, Vincenzina; Garcia-Martínez, Pedro; Jiménez-Vicario, Pedro.

8. El uso de rompecabezas en la enseñanza de la historia urbana. The use of puzzles in the teaching of urban history. Esteller-Agustí, Alfred; Vigil-de Insausti, Adolfo; Herrera-Piñuelas, Isamar Anicia.

9. Estrategias educativas innovadoras para la docencia teórica en Arquitectura. Innovative Educational Strategies for Theoretical Teaching in Architecture. LopezDe Asiain, María; Díaz-García, Vicente.

10. Los retos de la evaluación online en el aprendizaje universitario de la arquitectura. Challenges of online evaluation in the Architecture University learning. Onecha-Pérez, Belén; López-Valdés, Daniel; Sanz-Prat, Javier. 
11. Zoé entra en casa. La biología en la formación en arquitectura. Zoé enters the house. Biology in architectural training. Tapia Martín, Carlos; Medina Morillas, Carlos.

12. Elementos clave de una base sólida que estructure la docencia de arquitectura. Key elements of a solid foundation that structures architectural teaching. Santalla-Blanco, Luis Manuel.

13. Buildings $360^{\circ}$ : un nuevo enfoque para la enseñanza en construcción. Buildings 360 : a new approach to teaching construction. Sánchez-Aparicio, Luis Javier; Sánchez-Guevara Sánchez, María del Carmen; Gallego Sánchez-Torija, Jorge; Olivieri, Francesca.

14. Asignaturas tecnológicas en Arquitectura en el confinamiento: hacia una enseñanza aplicada. Technological courses in Architecture during lock down: towards an applied teaching. Cornadó, Còssima; Crespo, Eva; Martín, Estefanía.

15. Pedagogía colaborativa y redes sociales. Diseñar en cuarentena. Collaborative Pedagogy and Social Networks. Design in Quarantine. Hernández-Falagán, David.

16. De Vitruvio a Instagram: Nuevas metodologías de análisis arquitectónico. From Vitruvius to Instagram: New methodologies for architectural análisis. Coeffé Boitano, Beatriz.

17. Estrategias transversales. El grano y la paja. Transversal strategies. Wheat and chaff. Alfaya, Luciano; Armada, Carmen.

18. Lo fortuito como catalizador para el desarrollo de una mentalidad de crecimiento. Chance as a catalyst for the development of a growth mindset. Amtmann-Barbará, Sebastián; Mosquera-González, Javier.

19. Sevilla: Ciudad Doméstica. Experimentación y Crítica Urbana desde el Confinamiento. Sevilla: Domestic City. Experimentation and Urban Critic from Confinement. Carrascal-Pérez, María F.; Aguilar-Alejandre, María.

20. Proyectos con Hormigón Visto. Repensar la materialidad en tiempos de COVID19. Architectural Design with Exposed Concrete. Rethinking materiality in times of COVID-19. Lizondo-Sevilla, Laura; Bosch-Roig, Luis.

21. El Database Driven Lab como modelo pedagógico. Database Driven Lab as a pedagogical model. Juan-Liñán, Lluís; Rojo-de-Castro, Luis.

22. Taller de visitas de obra, modo virtual por suspensión de docencia presencial. Building site visits workshop, virtual mode for suspension of in-class teaching. Pinilla-Melo, Javier; Aira, José-Ramón; Olivieri, Lorenzo; Barbero-Barrera, María del Mar. 
23. La precisión en la elección y desarrollo de los trabajos fin de máster para una inserción laboral efectiva. Precision in the choice and development of the final master's thesis for effective job placement. Tapia-Martín, Carlos; Minguet-Medina, Jorge.

24. Historia de las mujeres en la arquitectura. 50 años de investigación para un nuevo espacio docente. Women's History in Architecture. 50 years of reseach for a new teaching area. Pérez-Moreno, Lucía C.

25. Sobre filtros aumentados transhumanos. HYPERFILTER, una pedagogía para la acción FOMO. On transhuman augmented filters. HYPERFILTER, a pedagogy for FOMO Action. Roig, Eduardo.

26. El arquitecto ante el nuevo paradigma del paisaje: implicaciones docentes. The architect addressing the new landscape paradigm: teaching implications. LópezSanchez, Marina; Linares-Gómez, Mercedes; Tejedor-Cabrera, Antonio.

27. 'Arquigramers'. 'Archigramers'. Flores-Soto, José Antonio.

28. Poliesferas Pedagógicas. Estudio analítico de las cosmologías locales del Covid19. Pedagogical Polysoheres. Analytical study of the local cosmologies of the Covid-19. Espegel-Alonso, Carmen; Feliz-Ricoy, Sálvora; Buedo-García, Juan Andrés.

29. Académicas enREDadas en cuarentena. Academic mamas NETworking in quarantine. Navarro-Astor, Elena; Guardiola-Víllora, Arianna.

30. Aptitudes de juicio estético y visión espacial en alumnos de arquitectura. Aesthetic judgment skills and spatial vision in architecture students. Iñarra-Abad, Susana; Sender-Contell, Marina; Pérez de los Cobos-Casinello, Marta.

31. La docencia en Arquitectura desde la comprensión tipológica compositiva. Teaching Architecture from a compositive and typological understanding. Cimadomo, Guido.

32. Habitar el confinamiento: una lectura a través de la fotografía y la danza contemporánea. Inhabiting confinement: an interpretation through photography and contemporary dance. Cimadomo, Guido.

33. Docencia Conversacional. Conversational learning. Barrientos-Turrión, Laura.

34. ¿Arquitectura a distancia? Comparando las docencias remota y presencial en Urbanismo. Distance Learning in Architecture? Online vs. On-Campus Teaching in Urbanism Courses. Ruiz-Apilánez, Borja; García-Camacha, Irene; Solís, Eloy; Ureña, José María de. 
35. El taller de paisaje, estrategias y objetivos, empatia, la arquitectura como respuesta. The landscape workshop, strategies and objectives, empathy, architecture as the answer. Jiliberto-Herrera, José Luís.

36. Yo, tú, nosotras y el tiempo en el espacio habitado. Me, you, us and time in the inhabited space. Morales-Soler, Eva; Minguet-Medina, Jorge.

37. Mis climas cotidianos. Didácticas para una arquitectura que cuida el clima y a las personas. Climates of everyday life. Didactics for an Architecture that cares for the climate and people. Alba-Pérez-Rendón, Cristina; Morales-Soler, Eva; MartínRuíz, Isabel.

38. Aprendizaje confinado: Oportunidades y percepción de los estudiantes. Confined learning: Opportunities and perception of college students. Redondo-Pérez, María; Muñoz-Cosme, Alfonso.

39. Arqui-enología online. La arquitectura de la percepción, los sentidos y la energía. Archi-Oenology online. The architecture of senses, sensibilities and energies. Ruiz-Plaza, Angela.

40. La piel de Samantha: presencia y espacio. Propuesta de innovación docente en Diseño. The skin of Samantha: presence and space. Teaching innovation proposal in Design. Fernández-Barranco, Alicia.

41. El análisis de proyectos como aprendizaje transversal en Diseño de Interiores. Analysis of projects as a transversal learning in Interior Design. González-Vera, Víctor Miguel; Fernández-Contreras, Raúl; Chamizo-Nieto, Francisco José.

42. El dibujo como herramienta operativa. Drawing as an operational tool. Bacchiarello, María Fiorella.

43. Experimentación con capas tangibles e intan-gibles: COVID-19 como una capa intangible más. Experimenting with tangible and intangible layers: COVID-19 as another intangible layer. Sádaba, Juan; Lenzi, Sara; Latasa, Itxaro.

44. Logros y Límites para una enseñanza basada en el Aprendizaje en Servicio y la Responsabilidad Social Universitaria. Achievements and Limits for teaching based on Service Learning and University Social Responsibility. Ríos-Mantilla, Renato; Trovato, Graziella.

45. Generación screen: habitar en tiempos de confinamiento. Screen Generation: Living in the Time of Confinement. De-Gispert-Hernández, Jordi; García-Ortega, Ramón.

46. Sobre el QUIÉN en la enseñanza arquitectónica. About WHO in architectural education. González-Bandera, María Isabel; Alba-Dorado, María Isabel. 
47. La docencia del dibujo arquitectónico en época de pandemia. Teaching architectural drawing in times of pandemic. Escoda-Pastor, Carmen; Sastre-Sastre, Ramon; Bruscato-Miotto Underlea.

48. Aprendizaje colaborativo en contextos postindustriales: catálogos, series y ensamblajes. Collaborative learning in the post-industrial context: catalogues, series and assemblies. de Abajo Castrillo, Begoña; Espinosa Pérez, Enrique; GarcíaSetién Terol, Diego; Ribot Manzano, Almudena.

49. El Taller de materia. Creatividad en torno al comportamiento estructural. Matter workshop. Creativity around structural behavior. Arias Madero, Javier; Llorente Álvarez, Alfredo.

50. Human 3.0: una reinterpretación contemporánea del Ballet Triádico de Oskar Schlemmer. Human 3.0: a contemporary reinterpretation of Oskar Schlemmer's Triadic Ballet. Tabera Roldán, Andrés; Vidaurre-Arbizu, Marina; Zuazua-Ros, Amaia; González-Gracia, Daniel.

51. ¿Materia o bit? Maqueta real o virtual como herramienta del Taller Integrado de Proyectos. Real or Virtual Model as an Integrative Design Studio Tool. TárragoMingo, Jorge; Martín-Gómez, César; Santas-Torres, Asier; Azcárate-Gómez, César.

52. Un estudio comparado. Hacía la implantación de un modelo docente mixto. $A$ comparative study. Towards the implementation of a mixed teaching model. Pizarro Juanas, María José; Ruiz-Pardo, Marcelo; Ramírez Sanjuán, Paloma.

53. De la clase-basílica al mapa generativo: Las redes colaborativas del nativo digital. From the traditional classroom to the generative map: The collaborative networks of the digital native. Martínez-Alonso, Javier; Montoya-Saiz, Paula.

54. Confinamiento liberador: experimentar con materiales y texturas. Liberating confinement: experimenting with materials and textures. De-Gispert-Hernández, Jordi.

55. Exposiciones docentes. Didáctica, transferencia e innovación en el ámbito académico. Educational exhibitions. Didacticism, transfer and innovation into the academic field. Domigo Santos, Juana; Moreno Álvarez, Carmen; García Píriz, Tomás.

56. Comunicacción. Acción formativa sobre la comunicación efectiva. Communicaction. Training action about the effective communication. Rivera, Rafael; Trujillo, Macarena.

57. Oscilación entre teoría y práctica: la representación como punto de equilibrio. Oscillation between theory and practice: representation as a point of balance. Andrade-Harrison, Pablo. 


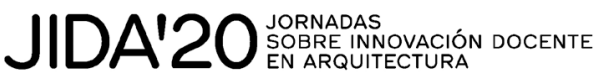

58. Construcción de Sentido: Rima de Teoría y Práctica en el Primer Año de Arquitectura. Construction of Meaning: Rhyme of Theory and Practice in the First Year of Architecture. Quintanilla-Chala, José; Razeto-Cáceres, Valeria.

59. Propuesta innovadora en el Máster Oficial en Peritación y Reparación de Edificios. Innovative proposal in the Official Master in Diagnosis and Repair of Buildings. Pedreño-Rojas, Manuel Alejandro; Pérez-Gálvez, Filomena; MoralesConde, María Jesús; Rubio-de-Hita, Paloma.

60. La inexistencia de enunciado como enunciado. The nonexistence of statement as statement. García-Bujalance, Susana.

61. Blended Learning en la Enseñanza de Proyectos Arquitectónicos a través de Miro. Blended Learning in Architectural Design Education through Miro. CoelloTorres, Claudia.

62. Multi-Player City. La producción de la ciudad negociada: Simulaciones Docentes. Multi-Player City. The production of the negociated city: Educational Simulations. Arenas Laorga, Enrique; Basabe Montalvo, Luis; Muñoz Torija, Silvia; Palacios Labrador, Luis.

63. Proyectando un territorio Expo: grupos mixtos engarzando el evento con la ciudad existente. Designing an Expo space: mixed level groups linking the event with the existing city. Gavilanes-Vélaz-de-Medrano, Juan; Castellano-Pulido, Javier; Fuente-Moreno, Jesús; Torre-Fragoso, Ciro.

64. Un pueblo imaginado. An imagined village. Toldrà-Domingo, Josep Maria; FarrenyMorancho, Jaume; Casals-Roca, Raquel; Ferré-Pueyo, Gemma.

65. El concurso como estrategia de aprendizaje: coordinación, colaboración y difusión. The contest as a learning strategy: coordination, collaboration and dissemination. Fernández Villalobos, Nieves; Rodríguez Fernández, Carlos; Geijo Barrientos, José Manuel.

66. Aprendizaje-Servicio para la diagnosis socio-espacial de la edificación residencial. Service-Learning experience for the socio-spatial diagnosis of residential buildings. Vima-Grau, Sara; Tous-Monedero, Victoria; Garcia-Almirall, Pilar.

67. Creatividad con método. Evolución de los talle-res de Urbanismo+Proyectos de segundo curso. Creativity within method. Evolution of the se-cond year Architecture+Urban design Studios. Frediani Sarfati, Arturo; Alcaina Pozo, Lara; Rius Ruiz, Maria; Rosell Gratacòs, Quim.

68. Estrategias de integración de la metodología BIM en el sector AEC desde la Universidad. Integration strategies of the BIM methodology in the AEC sector from the University. García-Granja, María Jesús; de la Torre-Fragoso, Ciro; BlázquezParra, Elidia B.; Martin-Dorta, Norena. 
69. Taller experimental de arquitectura y paisaje. Primer ensayo "on line". Architecture and landscape experimental atelier. First online trial. Coca-Leicher, José de; Fontcuberta-Rueda, Luis de.

70. camp_us: co-diseñando universidad y ciudad. Pamplona, 2020. camp_us: codesigning university and city. Pamplona 2020. Acilu, Aitor; Larripa, Adrián.

71. Convertir la experiencia en experimento: La vida confinada como escuela de futuro. Making the experience into experiment: daily lockdown life as a school for the future. Nanclares-daVeiga, Alberto.

72. Urbanismo Acción: Enfoque Sostenible aplicado a la movilidad urbana en centros históricos. Urbanism Action: Sustainable Approach applied to urban mobility in historic centers. Manchego-Huaquipaco, Edith Gabriela; Butrón-Revilla, Cinthya Lady.

73. Arquitectura Descalza: proyectar y construir en contextos frágiles y complejos. Barefoot Architecture designing and building in fragile and complex contexts. López-Osorio, José Manuel; Muñoz-González, Carmen M.; Ruiz-Jaramillo, Jonathan; Gutiérrez-Martín, Alfonso.

74. I Concurso de fotografía de ventilación y climatización: Una experiencia en Instagram. I photography contest of ventilation and climatization: An experience on Instagram. Assiego-de-Larriva, Rafael; Rodríguez-Ruiz, Nazaret.

75. Urbanismo participativo para la docencia sobre espacio público, llegó el confinamiento. Participatory urbanism for teaching on public space, the confinement arrived. Telleria-Andueza, Koldo; Otamendi-Irizar, Irati.

76. WhatsApp: Situaciones y Programa. WhatsApp: Situations and Program. Silva, Ernesto; Braghini, Anna; Montero Paulina.

77. Los talleres de experimentación en la formación del arquitecto humanista. The experimental workshops in the training of the humanist architect. DomènechRodríguez, Marta; López López, David.

78. Role-Play como Estrategia Docente en el Aprendizaje de la Construcción. RolePlay as a Teaching Strategy in Construction Learning. Pérez-Gálvez, Filomena; Pedreño-Rojas, Manuel Alejandro; Morales-Conde, María Jesús; Rubio-de-Hita, Paloma.

79. Enseñanza de la arquitectura en Chile. Acciones pedagógicas con potencial innovador. Architectural teaching in Chile. Pedagogical actions with innovative potential. Lagos-Vergara, Rodrigo; Barrientos-Díaz, Macarena. 
80. Taller vertical y juego de roles en el aprendizaje de programas arquitectónicos emergentes. Vertical workshop and role-playing in the learning of emerging architectural programs. Castellano-Pulido, F. Javier; Gavilanes-Vélaz de Medrano, Juan; Minguet-Medina, Jorge; Carrasco-Rodríguez, Francisco.

81. Un extraño caso de árbol tenedor. Madrid y Ahmedabad. Aula coopera [Spain/in/India]. A curious case of tree fork. Madrid and Ahmedabad. Aula coopera [Spain/in/India]. Montoro-Coso, Ricardo; Sonntag, Franca Alexandra.

82. La escala líquida. Del detalle al territorio como herramienta de aprendizaje. Liquid scale. From detail to territory as a learning tool. Solé-Gras, Josep Maria; TiñenaRamos, Arnau; Sardà-Ferran, Jordi.

83. Empatía a través del juego. La teoría de piezas sueltas en el proceso de diseño. Empathy through playing. The theory of loose parts in Design Thinking. CabreroOlmos, Raquel.

84. La docencia de la arquitectura durante el confinamiento. El caso de la Escuela de Valencia. Teaching architecture in the time of stay-at-home order. The case of the Valencia School. Cabrera i Fausto, Ivan; Fenollosa Forner, Ernesto.

85. Proyectos Arquitectónicos de programa abierto en lugares invisibles. Architectural Projects of open program in invisible places. Alonso-García, Eusebio; Blanco-Martín, Javier. 


\title{
Arquigramers Archigramers
}

\author{
Flores-Soto, José Antonio
}

Departamento de Composición Arquitectónica, Escuela Técnica Superior de Arquitectura, Universidad Politécnica de Madrid, España. joseantonio.flores@upm.es

\begin{abstract}
Archigramers is a proposal that investigates the incorporation of the social network Instagram in the teaching of the subject 'Introduction to architecture', of the first year of the Degree in Fundamentals of Architecture at Etsam. The challenge of the subjects in the area of Architectural Composition, the theoretical ones of the career, is to know how to convey to the student the lesson of history and the value of the idea in the architectural project. The added challenge with a digital native generation is to incorporate into teaching, in a critical way, the digital tools that today's students use to access knowledge, very different from those conventionally used in this type of subject: mainly books. This communication tells of the experience of the 'architect's notebook' as a tool for thinking about architecture, turned into a digital tool through the use of Instagram.
\end{abstract}

Keywords: theory, ICT tools, theory and analysis, architectural composition, introduction to architecture.

Thematic areas: theory, TIC tools, theory and analysis.

\begin{abstract}
Resumen
Arquigramers es una propuesta que indaga en la incorporación de la red social Instagram en la docencia de la asignatura Introducción a la arquitectura, de primer curso del Grado en Fundamentos de la Arquitectura de la Etsam. El reto de las asignaturas del área de Composición Arquitectónica, las teóricas de la carrera, es saber trasladar al estudiante la lección de la historia y el valor de la idea en el proyecto arquitectónico. El reto añadido con una generación nativa digital es incorporar a la docencia, de manera crítica, las herramientas digitales que los estudiantes de hoy manejan para acceder al conocimiento, muy distante de las convencionalmente usadas en este tipo de asignaturas: libros principalmente. Esta comunicación cuenta la experiencia del 'cuaderno del arquitecto' como herramienta para pensar arquitectura, convertido en una herramienta digital a través del uso de Instagram.
\end{abstract}

Palabras clave: teoría, herramientas TIC, teoría y análisis, composición arquitectónica, introducción a la arquitectura.

Bloque temático: teoría, herramientas TIC, teoría y análisis. 


\section{Introducción}

Con casi dos décadas de trayectoria vital, las redes sociales son coetáneas de los estudiantes que llenan hoy las aulas universitarias (Alemany Martínez, 2009). Se puede asegurar que los estudiantes que ingrersan en las Escuelas de Arquitectura desde hace unos años son nativos digitales; no así los docentes que han de encargarse de ellos. Así pues, la experiencia y visión que tienen del mundo en que están inmersos, no sólo de la actividad arquitectónica a la que han dirigido sus pasos, depende en gran medida de ellas. Los estudiantes de ingreso reciente en la universidad están tan habituados al uso de redes sociales en sus cotidianidades, que ni siquiera se planteen cómo era la vida antes de que existiesen Twitter, Facebook o Instagram; es algo que no han vivido, carecen de esa experiencia de la que sí participan quienes les imparten docencia. Por el mismo motivo, a la gran mayoría de ellos los medios escritos de difusión en papel: libros y revistas, tradicionales fuentes de difusión del conocimiento en las áreas más teóricas de la carrera, les resultan obsoletos; no suelen tener el hábito de su uso y consulta como sí están acostumbrados a ello sus docentes. Y esto mismo sucede con las herramientas de análisis y expresión: lo manual, lo físico no es su mundo habitual. Esta diferencia generacional en el acceso a las fuentes solventes de información y en el uso de herramientas de indagación y conocimiento es un campo sugerente de experimentación e investigación y, sin duda, de reflexión. No en vano la cuestión es cómo transmitir el conocimiento de la manera más efectiva y, como dijera Ortega y Gasset, enseñar a los estudiantes a pensar a partir de esa transferencia producida en las aulas (Ortega y Gasset, 1930).

Mientras que los docentes (en torno a la treintena los más jóvenes) procenden de un modo de hacer donde los libros y demás medios de difusión escrita e impresa son la principal fuente de consulta y de difusión del conocimiento, los estudiantes que comienzan su andadura universitaria (en casi su totalidad con 18 años) tienen internet como primera fuente de búsqueda de información (Moreno Moreno, 2018); para ellos primero es internet y luego, en un papel secundario, son los libros. Parece pertinente, pues, una reflexión sobre el acercamiento de estas dos experiencias, como proceso crítico para ofrecer una mejor solución a la cuestión de la formación en una disciplina que, como la arquitectónica, está profundamente ligada con la generación de los espacios donde se desarrolla la vida. Si bien es cierto que en los aspectos más teóricos de esta disciplina las fuentes documentales escritas son y han sido esenciales, carece de sentido obviar los modos en que los estudiantes de hoy se aproximan al conocimiento. Al fin y al cabo, como apunta Richard Sennett (Sennett, 2008), la primera creación del 'hombre' es él mismo; y no sería muy realista una formación que olvidase por completo, por los evidentes riesgos que presenta, la manera en que se 'crean' a sí mismos y 'su mundo' (el que van a transformar mediante la arquitectura) quienes serán arquitectos mañana, estudiantes ahora.

Esta comunicación pretende una reflexión sobre la incorporación crítica del uso de las redes sociales a los métodos convencionales de transmisión del conocimento en el área de la Composición Arquitectónica. En la línea de las que se vienen produciendo en las JIDAs de estos últimos años, se propone una reflexión según el experimento docente efectuado con un grupo de estudiantes de primer curso de la Escuela de Arquitectura de Madrid. El caso concreto fue el de la incorporación de la red social Instagram como herramienta de reflexión y difusión de conocimiento, durante el curso académico 2019-2020, en la asignatura Introducción a la arquitectura, primera del 'Grado en Fundamentos de la Arquitectura'. 


\section{Introducción a la arquitectura, la primera asignatura de la carrera}

No todas las escuelas de arquitectura españolas contemplan la necesidad de una asignatura introductoria que, con carácter general, ofrezca una visión preliminar de la disciplina a los estudiantes de primer curso. En el plan de estudios actualmente vigente en la Escuela de Arquitectura de Madrid para el Grado en Fundamentos de la Arquitectura (Plan 2010), sí se incluye Introducción a la arquitectura como asignatura cuyo cometido primordial es presentar la disciplina arquitectónica en sus conceptos fundamentales a quienes se aproximan por primera vez a ella. Desde el plano teórico propio del área de Composición Arquitectónica, de la cual es su primera materia, se aborda en ella el conocimiento de la arquitectura con un enfoque global. Se trata de una primera aproximación a lo arquitectónico que pretende ser al mismo tiempo atractiva y rigurosa. Esto implica enseñar a los estudiantes a acceder de forma clara y precisa a los conceptos básicos de la disciplina, en los que luego profundizarán otras asignaturas más específicas a lo largo del grado (Flores, 2019).

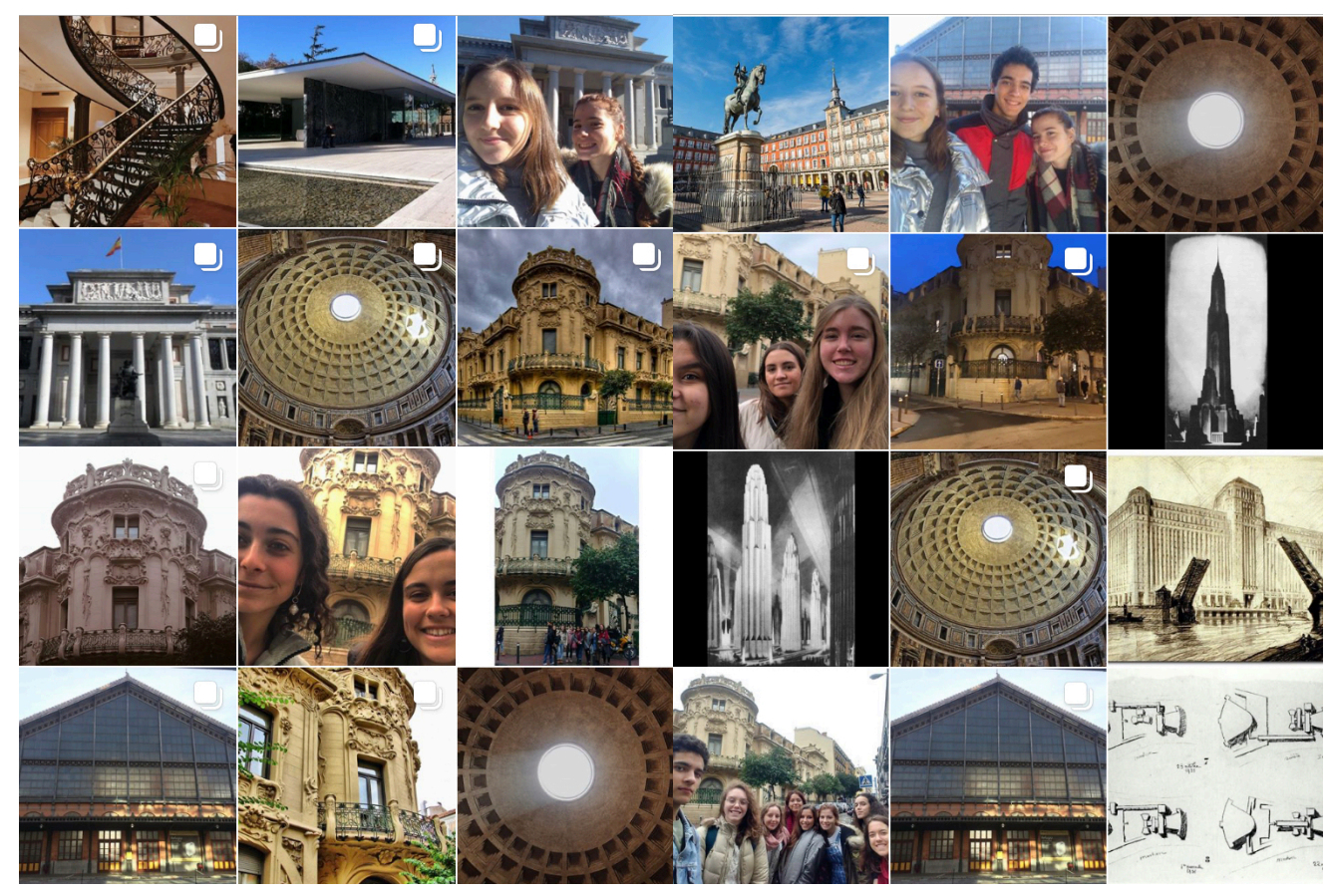

Fig.1 Introducción a la arquitectura, una nueva experiencia. Fuente: \#introetsamgrupof (Instagram, 2019)

Introducción a la arquitectura es, pues, la primera asignatura que los 'neófitos' se encuentran en la carrera. ${ }^{1}$ Gran parte de los estudiantes que la siguen lo hacen sin relación directa previa (académica o personal) con el ámbito de la arquitectura y es por ello que les centra en la elección que acaban de hacer en sus vidas universitarias. Se trata de una primera inmersión en las particularidades de la disciplina, una reflexión general sobre ella a través de conceptos básicos que le son propios y de ejemplos fundamentales que los representan, extraidos de entre todos los momentos históricos (generalmente de la cultura occidental), incluidos los años recientes. En ella, el papel del docente es primordial a la hora de la transferencia de conocimientos así como de la incitación a ciertos 'modos de hacer' propios del arquitecto. A él le toca ejercer de singular

\footnotetext{
${ }^{1}$ De hecho, por cuestiones de organización horaria, para cuatro de los ocho grupos que tiene el primer curso del 'Grado en Fundamentos de la Arquitectura' en la ETSAM, Introducción a la arquitectura es literalmente la primera asignatura que se encuentran porque se imparte a primera hora del turno. Para los otros cuatro grupos, en cambio, es Cálculo, del Dpto. de Matemática Aplicada.
} 
y primer guía y apoyo en este arranque; de modo que de alguna manera su tarea es señalar el camino por el que han de transitar luego en los largos años de carrera: les eseña a mirar, en términos del profesor Campo Baeza.

Generalmente uno de los problemas en la docencia de esta asignatura introductoria se encuentra en la cantidad y densidad de conceptos teóricos que han de trasladarse al alumnado neófito, que ni siquiera ha cursado en el bachillerato una asignatura de historia del arte donde mínimamente se les familiarice con la disciplina arquitectónica. ${ }^{2}$ El otro problema es el tiempo brevísimo en que esta transmisión de conocimiento ha de hacerse, dada la estructura cuatrimestral del plan de estudios. Se podría decir que son dos problemas consustanciales a todo el Grado, pero el hecho de ser la primera vez que se enfrenta al estudiante a ello hace que el efecto sea más traumático por impactante (luego se acaban acostumbrando al ritmo de vértigo y se olvidan quizás de este arduo comienzo). Así pues, se está ante un alumnado al que hay que presentar una disciplina a la que son mayoritariamente ajenos; y hay que hacerlo, a grandes rasgos y con rigor, a través de conceptos teóricos en su mayor parte novedosos para ellos y mediante herramientas a las que no suelen estar familiarizados. Esto se produce en tiempo muy escaso, en compañía de otras asignaturas más específicas: generalmente gráficas, y con grandes dificultades para que se dé un mínimo periodo de reflexión y madurez para la asimilación de la información recibida. La cuestión es que esta primera avalancha de información se haga entonces con tanto rigor como atractivo, si no para que sea menor la carga, al menos para que no les resulte tan ajena y para atraparlos en el interés por hacer arquitectura, que es a lo que mayoritariamente dicen venir a aprender y a lo que quieren dedicar sus vidas profesionales. ${ }^{3}$

En este contexto de flujo vertiginoso de información, parece tarea del docente aproximarse lo más posible a los modos de hacer y de comprender el mundo de su auditorio. No tendría sentido encastillarse sólo en los métodos tradicionales de la clase magistral, las referencias bibliográficas y las visitas, obviando el amplio mundo de internet y de las redes sociales tan propio de los estudiantes de ahora. Pero tampoco esventregarse acríticamente a los medios disponibles en la red, sino de tratar de incorporarlos en beneficio de los contenidos a impartir y de una mejor relación entre el conocimiento académico y el mundo actual y sus particularidades.

\section{El contexto de otras experiencias}

La profesora Carmen Díez Medina reflexionaba en las VI JIDAs sobre las particularidades en la docencia del área de Composición Arquitectónica, fudamentalmente relacionada con conceptos teóricos que pudieran resultar obsoletos a estudiantes actuales ante un mundo en constante cambio. Uno de los retos de la docencia actual en este ámbito, con estudiantes acostumbrados al ritmo vertiginoso de obsolescencia de la realidad que les es cotidiana, es hacerles comprender la validez de la lección de la historia en sus ejemplos más significativos (Díez Medina, 2018). Casi parafraseando al profesor y arquitecto Alberto Campo Baeza, la profesora Díez Medina recordaba entonces que la arquitectura se construye con ideas (Campo Baeza, 1996) -la buena arquitectura- y que éstas se han de enseñar necesariamente en las escuelas. Ideas; de eso se trata en la docencia de Introducción a la arquitectura: de enseñar las ideas que han fundamentado las arquitecturas a lo largo de la historia para hacer ver que también las ha de

\footnotetext{
${ }^{2}$ Los estudiantes de nuevo ingreso en el Grado de Fundamentos de la Arquitectura suelen proceder del bachillerato técnico.

${ }^{3}$ Afirmaciones fundamentadas en el análisis de los datos de la encuesta que se les pasa a los alumnos en el primer día de clase del grupo en cuestión, inéditos por ser material de trabajo. En esta encuesta el 95\% de los estudiantes manifestó querer ser arquitecto para proyectar y construir edificios.
} 
haber como soporte de la arquitectura de ahora. Enseñar y hacer reflexionar a los estudiantes sobre el hecho de que no hay arquitectura sin ideas detrás, de que el ejercicio de la arquitectura es intelectual, social, artístico (tan complejo como la sociedad a la que da soporte espacial), es tarea más que reseñable de esta asignatura introductoria con la esperanza de que se abude en ello en las siguientes más específicas.

Análisis, reflexión, dibujo, comparación, argumentación son herramientas que la profesora Díez Medina empleaba entonces y que se juzgan apropiadas para el fin fundamental de enseñar a los recién ingresados estudiantes que la arquitectura se construye (siempre lo ha hecho) con fundamento en ciertas ideas. Precisamente algo de ese enfoque es el que ha animado la docencia del grupo cuya experiencia se transmite en esta comunicación, desde el principio enunciado por Bruno Zevi de pensar la arquitectura a través del dibujo y a través de la experiencia de la propia arquitectura (Zevi, 1951).

Respecto al empleo de TICs en la docencia de la disciplina arquitectónica varias han sido las aportaciones en estas JIDAs, con especial incidencia desde el grupo de investigación de la Universidad de Málaga, donde trabaja la profesora María Isabel Alba Dorado (Alba Dorado, 2018 y 2019). Si los conceptos fundamentales que se refieren a las ideas sobre las que se construye la arquitectura, a las que se referería la profesora Díez Medina, se transmiten a través de los libros principalmente $y$, sin embargo, parece conveniente acercarse al empleo de herramientas TIC de las que son usuarios los estudiantes, como indica la profesora Alba Dorado, la cuestión es cómo vincular ambos ámbitos de manera crítica y efectiva.

Contaba el profesor José Ramón Alonso Pereira ${ }^{4}$ en las IV JIDAs sus experiencias con el empleo de TICs en la docencia de esta asignatura introductoria en la Escuela de Arquitectura de La Coruña (Alonso Pereira, 2016). En su caso, la incorporación de blogs de temática arquitectónica que potenciasen la interacción entre estudiantes y docentes planteaba una reflexión sobre el debate intergeneracional entre ambos grupos. Esta labor de prensar la arquitectura entre docentes y estudiantes, en un medio abierto además al público, pretendía una mayor implicación y presencia exterior del debate fuera del ámbito de las aulas. $Y$ ese detalle de la exposición pública parece un dato relevante a tener en cuenta, pues es el medio en el que los estudiantes de ahora se desempeñan con total soltura en su cotidianidad a través de las redes sociales, que suponen un potente escaparate abierto al mundo donde mostrar sus vidas e intereses (o sus vidas e intereses convertidas en objeto de visulización).

En cuanto a experiencias con cierta semejanza a la que se pasará a exponer seguidamente, ya relatadas en las JIDAs, es necesario citar las de la profesora María Pura Moreno Moreno y las del profesor Jonathan Ruiz Jaramillo, en las Escuelas de Arquitectura de Cartagena y Málaga respectivamente. En el primer caso, desde el área de Proyectos Arquitectónicos (Moreno Moreno, 2018) el empleo de las herramientas TIC se centraba en el uso de la red social Instagram como complemento de reflexión, crítica y debate sobre las propuestas de trabajo en el taller de Proyectos I. En el segundo caso, cabe resaltar la investigación del uso de las TICs no tanto en el área de Composición Arquitectónica como también en el de Proyectos, a través de un 'cuaderno digital del arquitecto', donde se recogía y exponía al público el proceso de ideas para la génesis de los proyectos arquitectónicos (Ruiz Jaramillo, 2016). En ambos casos, la relfexión y el debate con argumentación eminentemente gráfica tenía la componente de exposición pública a través del uso de la red social Instagram, cuya principal característica es la de ser escaparate,

\footnotetext{
${ }^{4}$ Precisamente el profesor José Ramón Alonso Pereira es el catedrático de Introducción a la Arquitectura, en la Escuela de Arquitectura de La Coruña; otra de las escasas escuelas que incluyen en su plan de estudios la asignatura introductoria que se trata aquí.
} 
ventana abierta al munto 2.0 en el que tienen una vida paralela los estudiantes de hoy en día, como la generalidad de los jóvenes de la generación a la que pertenecen.

Ya que los estudiantes consumen en sus vidas cotidianas esta red social para mostrarse al mundo y comunicarse con otros como ellos, ¿por qué no aprovecharla para mostrar también el proceso de su reflexión creativa en el campo de la arquitectura en que se están formando? Las experiencias de los profesores Moreno Moreno y Ruiz Jaramillo desde el ámbito de la proyectación arquitectónica bien podrían hilarse con una similar desde el de la reflexión teórica que lo soporta; es decir, desde aquel otro en el que se enseñan las ideas que subyacen en el hacer arquitectónico, que es lo que se pretende transmitir en el área de Composición Arquitectónica. Por esta vía transita la experiencia de la propuesta 'Arquigramers' que aquí se presenta.

\section{Mirar para ver: el 'cuaderno del arquitecto'}

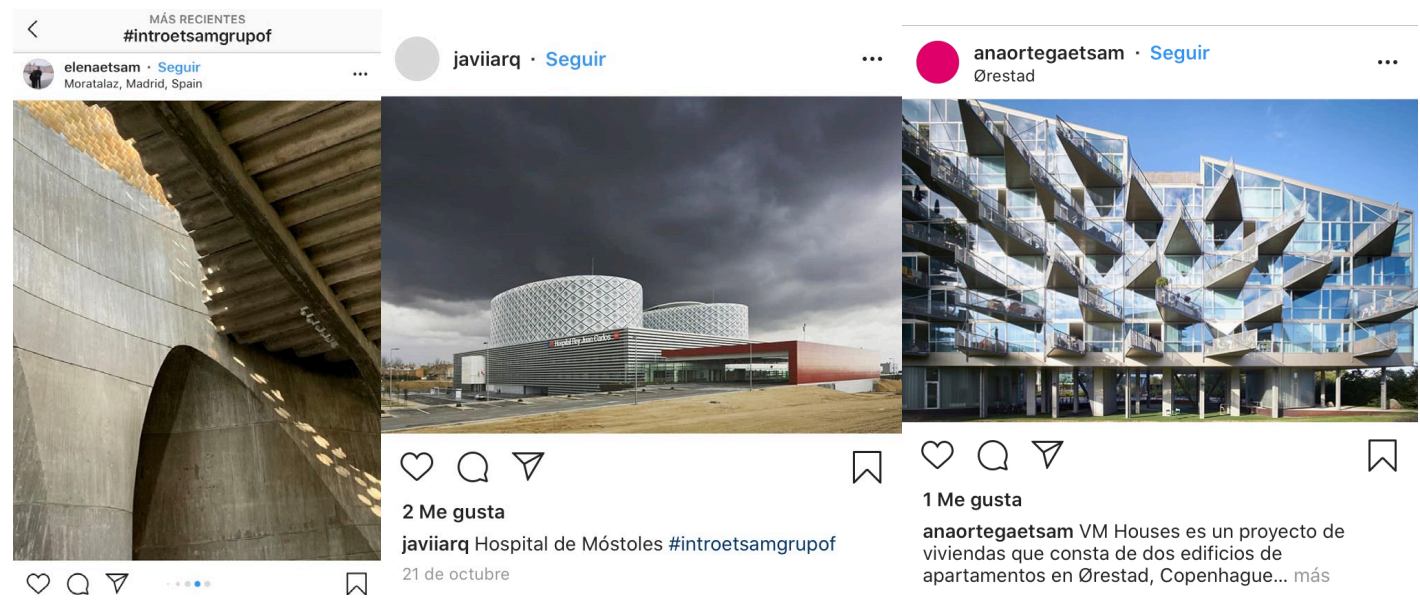

Fig.2 Ejemplo de ejercicio de clase en el cuaderno digital del arquitecto: \#introetsamgrupof

\subsection{El cuaderno como herramienta gráfica de pensamiento}

Si la arquitectura no es porque sí, sino que tiene tras de ella ideas que la sustentan, la principal tarea de un docente que se enfrenta a grupos de estudiantes recién ingresados en la escuela de arquitectura es enseñarles a ver arquitectura: mirar la arquitectura para ver tras ella las ideas que la generan y servirse de ellas para hacer arquitectura ahora. Ver arquitectura requiere tener los ojos bien abiertos predispuestos a analizar y escudriñar con intención lo que se mira con el nada desdeñable objetivo de aprender algo útil de ello. No en vano es máxima del profesor Félix de Azúa que la mirada no es innata en el ser humano, sino educada. ${ }^{5} \mathrm{~A}$ mirar se aprende con el ejercicio y con el tiempo: aprender a mirar para saber ver, dice el profesor Ximo Company cuando habla de la mejor manera de enfrentarse a la pintura de Velázquez (Company, 2018); lo que es trasladable a cualquier arquitectura de la que se pueda aprender algo de interés cuando se trata de enseñar a estudiantes recién ingresados en la Escuela de Arquitectura.

Pero la mirada, para aprender y que sea útil lo aprendido, se ha de apoyar en registros o apuntes donde almacenar aquello interesante que mirar: mirar para ver, fijar lo mirado en apuntes gráficos para aprender y estar luego en mejor disposición para hacer arquitectura. Por eso desde este

\footnotetext{
${ }^{5}$ Los Seminarios en el Museo del Prado. "El ojo que piensa”, que el profesor Azúa ha dirigido en los últimos años dan constancia de ello.
} 
grupo de Introducción a la arquitectura se hace especial hincapié en el 'cuaderno del arquitecto' como herramienta de reflexión, indagación y análisis de las ideas que sustentan los mejores ejemplos de arquitectura de todas las épocas. El 'cuaderno del arquitecto' hace uso, para el aprendizaje del neófito, de todo la enorme carga visual propia de la discipina. Es un registro gráfico donde el estudiante se ha de valer de todas aquellas herramientas propias del lenguaje arquitectónico para fijar conceptos propios de la disciplina: espacio, masa, piel, relación con el suelo, relación interior-exterior, contenido simbólico, relación forma-cometido, etc. a través de ejemplos que los materializan. El dibujo y la fotografía son el apoyo; la ciudad, los edificios (de cualquier época) son los casos de estudio; la lección del profesor Steen Eiler Rasmussen, la guía (Rasmussen, 2004 y 2013).

Al ser una asignatura introductoria y de carácter general ésta, la herramienta empleada del 'cuaderno del arquitecto' tiene también un sentido de iniciación. Persigue introducir al estudiante en el acercamiento a la arquitectura y la reflexión sobre sus valores más significativos. No se persigue tanto el resultado final como el hábito: de lo que se trata es de crear hábito en el mirar para ver, de hacer que el estudiante se familiarice con la arquitectura y con las herramientas de pensamiento propias de la disciplina, eminentemente gráficas. Por supuesto no se espera un resultado inmediato, pues lo importante no es el cuaderno en sí, sino la iniciación en una manera de enfrentarse a lo arquitectónico. No se trata de un cuaderno de proyección, como los mencionados de las experiencias previas de las escuelas de Cartagena y Málaga, sino un cuaderno de reflexión de ideas arquitectónicas. No obstante, se juzga una herramienta indispensable para la posterior faceta de proyección arquitectónica, pues incide en un método de pensar para luego hacer arquitectura.

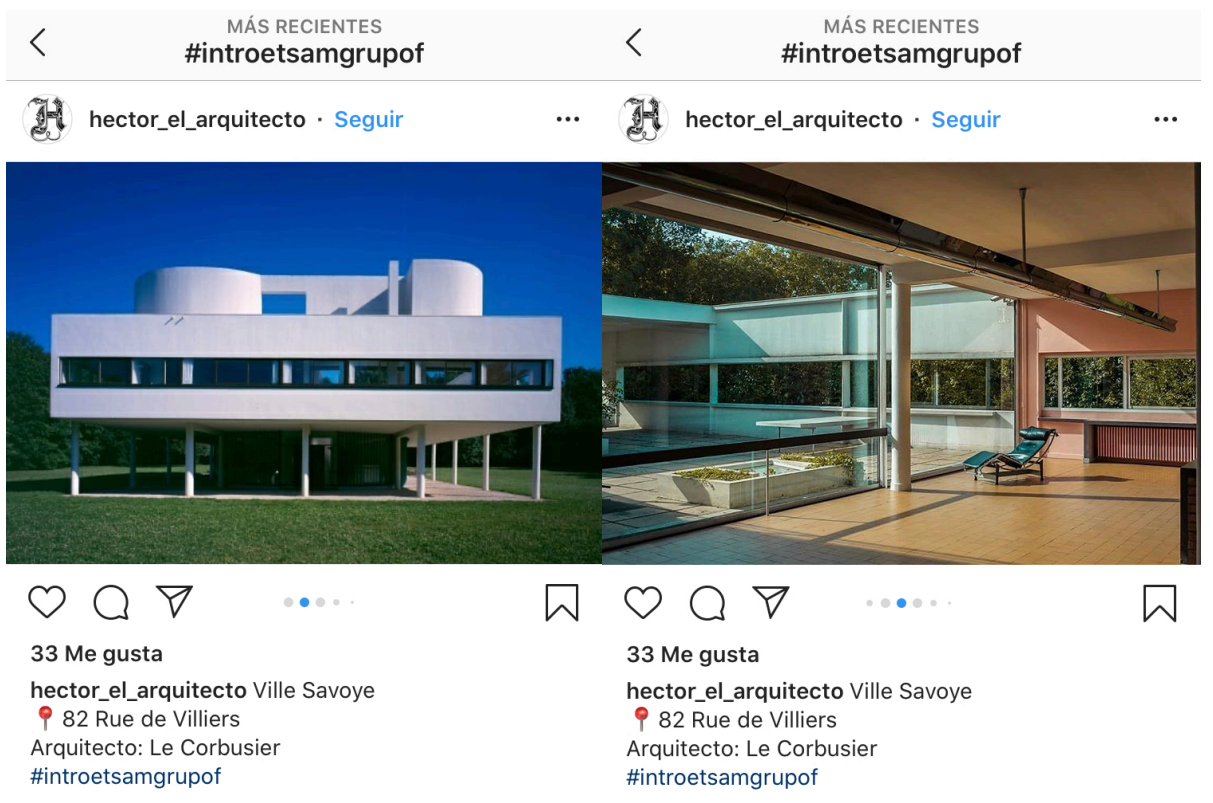

Fig.3 Ejemplo de ejercicio de clase en el cuaderno digital del arquitecto: villa Saboya: masa-espacio. Fuente: \#introetsamgrupof (Instagram, 2019)

\subsection{Instagram y el cuaderno digital del arquitecto}

Los estudiantes de nuevo ingreso no están tan habituados como hace años a los medios físicos, son nativos digitales. Mayoritariamente tienen internet como fuente primaria de consulta y las 
herramientas digitales como relación imporante con el conocimiento. Así pues, en esta experiencia se ha probado a incorporar aquellos medios digitales que son más habituales entre los estudiantes para transformar la idea del 'cuaderno del arquitecto' en una herramienta digital que se asemeje en parte a los usos que ellos le dan en sus vidas fuera de la Escuela. A los objetivos ya mencionados de propiciar en el estudiante una reflexión sobre la arquitectura, se añaden los de incorporar las posibilidades de visibilidad pública e interactuación que permiten los medios digitales: y esos medios son las redes sociales.

Desde el inicio se pensó que el 'cuaderno del arquitecto', en su versión digital debía de ser algo personal a la vez que estar abierto a aportar información para su visualización pública. La elección de la red social Instagram como soporte de esta experiencia tuvo en cuenta la familiaridad con que la tratan los propios estudiantes en su vida cotidiana, como ya se ha puesto de manifiesto en otras experiencias previas (Moreno Moreno, 2018). Instagram permite abrir un registro gráfico personal para cada estudiante a la vez que la interacción con otros estudiantes del grupo y con contactos ajenos al ámbito académico: amigos del instituto, del barrio o de vacaciones o familiares. De hecho, Instagram es una red social que los estudiantes ya usan mayoritariamente como escaparate público de sus actividades. ¿Por qué no incorporarla a su nueva experiencia académica como estudiantes de arquitectura? Del mismo modo que es una ventana abierta al mundo a través de la que se muestran como 'producto' social, podría verse como una ventana en la que mostrar su nueva faceta de estudiantes de arquitectura a la vez que convertirse en una herramienta digital para pensar la arquitectura que comienzan a descubrir.

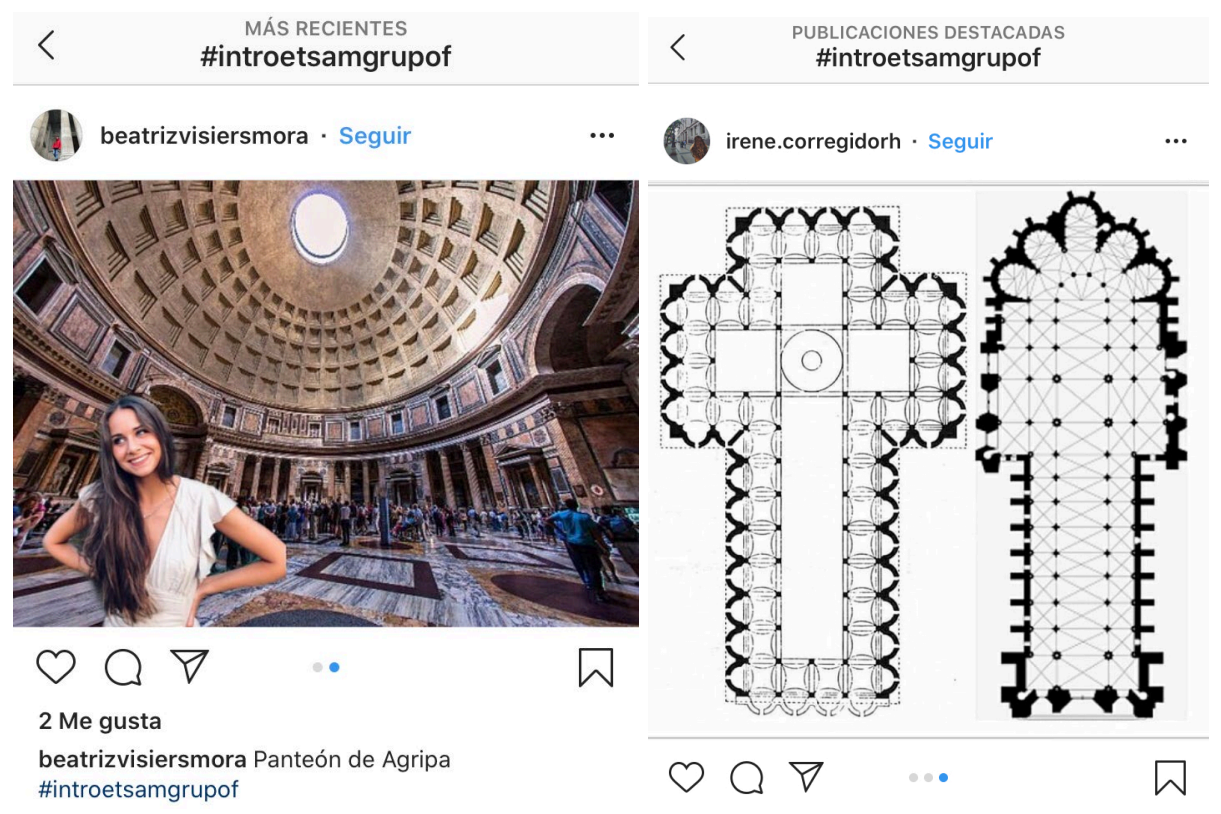

Fig.4 Ejemplo de ejercicio de clase en el cuaderno digital del arquitecto: Panteón, Espacio gótico-renacentista. Fuente: \#introetsamgrupof (Instagram, 2019)

No se pretendió generar una acumulación de referencias gráficas encontradas en la red archivadas por temas, como permite la red social Pinterest ya empleada en otras experiencias similares como la relatada por el profesor Ruiz Jaramillo (Ruiz Jaramillo, 2016). Y en este aspecto cabe mencionar el archivo del profesor Eduardo Pesquera en Pinterest (Pesquera, 2019), que se recomendó a los estudiantes como referencia. Esta red social, empleada con criterio sirve 
como un buen sistema de archivo de referencias web y permite su ordenación por temas. No obstante, no contempla el comentario ni la interacción del estudiante con la realidad arquitectónica. Y en este punto es preciso apuntar que el 'cuaderno del arquitecto' no sólo persigue la reflexión crítica sobre la arquitectura que se pueda encontrar reproducida gráficamente en la red, sino que pretende potenciar en lo posible la experiencia de la arquitectura. Ya decía Bruno Zevi que la representación de la arquitectura, aún para el nada desdeñable fin de conocerla mejor, no sustituye la experiencia de la propia arquitectura (Zevi, 1951). Así pues, siempre que los modelos de estudio lo han permitido, se ha optado por propiciar su experiencia directa para evitar que fuese un conocimiento exclusivamente documental.

Así pues, cada estudiantes se abrió una cuenta pública en Instagram, independiente de las propias que ya usasen si así lo consideraban oportuno, para emplearla como soporte de un 'cuaderno digital del arquitecto'. En ella debían subir periódicamente imágenes de los ejercicios semanales de clase, donde se analizaban aspectos muy concretos de los diversos ejemplos arquitectónicos estudiados. Además, sin olvidar la componente expositiva de la red, debían subir también fotografías de sus visitas a los edificios propuestos como casos de estudio. De este modo, cada uno de ellos tendría archivados cronológicamente sus ejercicios y visitas, visibles además para el público general y suscepctible de aportaciones en forma de comentarios.

La conexión entre todos los estudiantes del grupo de la asignatura se hizo mediante una etiqueta común: \#introestsamgrupof, a través de la cual fuese posible recuperar la actividad conjunta del grupo a cualquiera que le pudiese intersar su actividad. De modo que se propició así una actividad individual de cada estudiante con su cuaderno a la vez que una vinculación grupal, tan pertinente en estudiantes que acaban de llegar a la Escuela y no se conocen entre ellos. Al finalizar el cuatrimestre, una selección de imágenes recuperadas de la etiqueta común se expuso en las Jornadas de Puertas Abiertas de la Escuela, como muestra de la actividad realizada por el grupo durante su paso por la asignatura, lo que sirvió además para dar visibilidad a la experiencia y potenciar más su sentido entre los participantes.

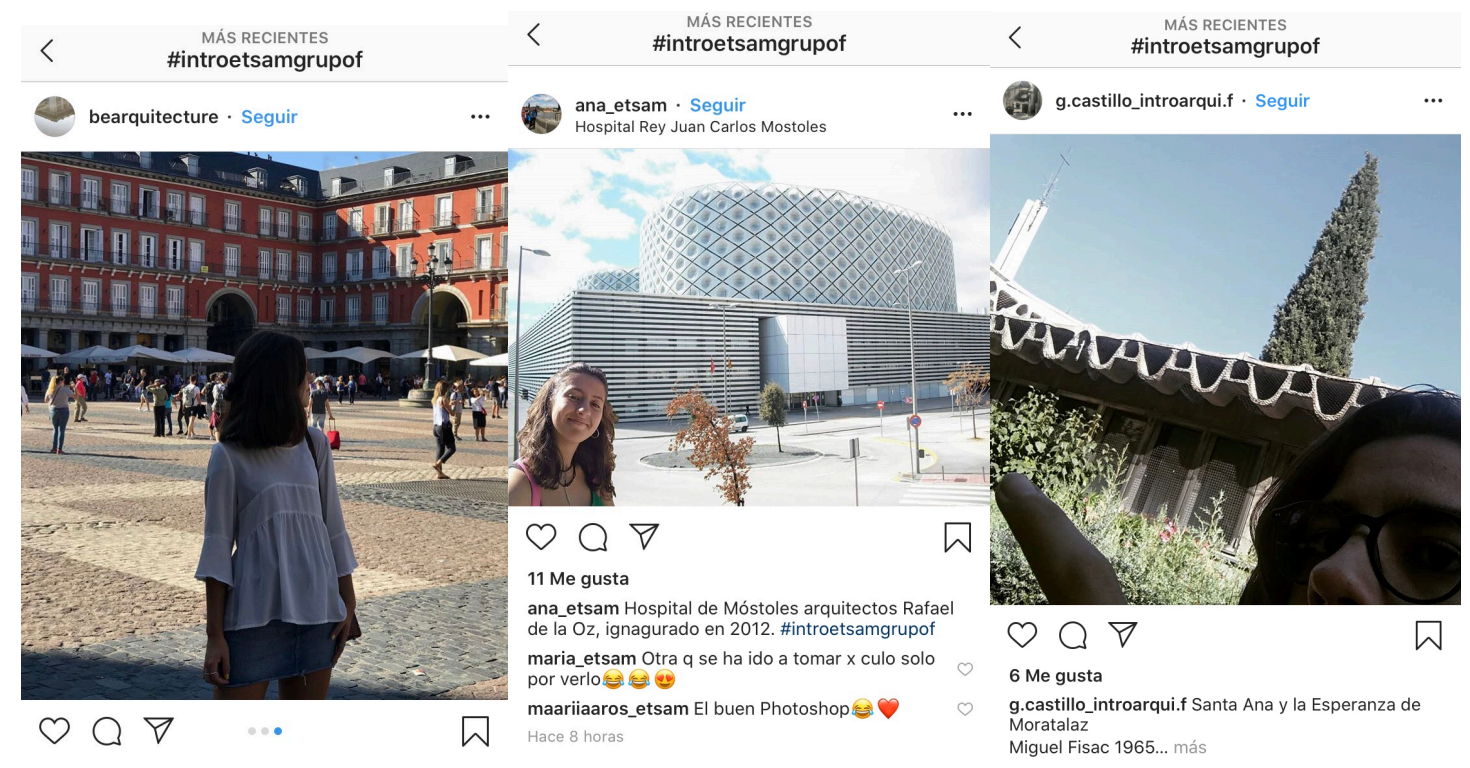

Fig. 5 Ejemplos de vistas (posibles e imposibles) a edificios y lugares. Fuente: \#introetsamgrupof (Instagram, 2019) 


\subsection{La versión del profesor: el relato paralelo de las arquitecturas dibujadas}

En paralelo a los cuadernos digitales del arquitecto de los estudiantes, también el docente participó en la experiencia con el suyo propio: @arquitecturasenpapel, abierto específicamente como complemento a esta experiencia docente experimental. Con la etiqueta común para las publicaciones del grupo: \#introestsamgrupof, en el cuaderno digital del profesor se ofreció a lo largo del curso un relato gráfico (inconcluso y activo para siguientes experiencias) sobre arquitectuas dibujadas de todo el espectro temporal, con preferencia por los tiempos recientes. Se trata de un lugar de referencia con el relato de arquitecturas que no han superado el estadio del papel, por tanto se han quedado en arquitecturas dibujadas.

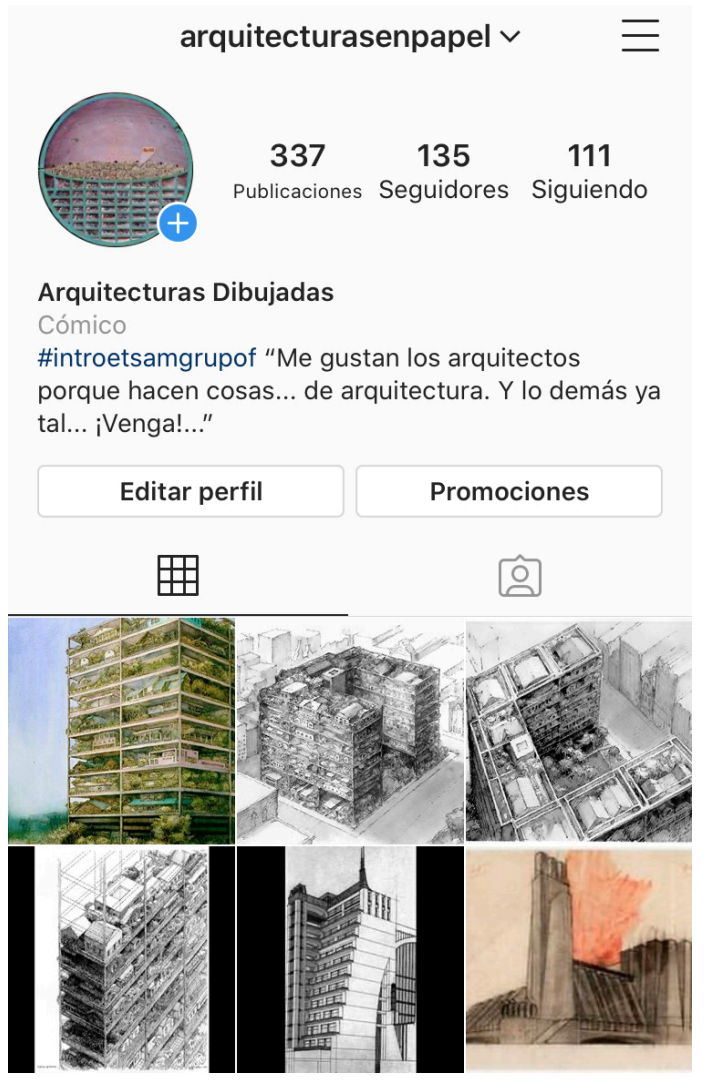

Fig.6 Perfil 'Arquitecturasenpapel' en Instagram

De este modo, se pretendió mostrar a los estudiantes que además de los ejemplos vistos y analizados en las clases presenciales ${ }^{6}$, también hay ideas interesantes en arquitecturas que se quedan en propuestas. Así pues, añadido al contenido de las clases del programa docente, este discurso gráfico sobre las arquitectuas dibujadas se suma la reflexión sobre ejemplos de los que puede aprender, con indicencia en determinados ejes temáticos.

\footnotetext{
${ }^{6}$ Al ser del primer cuatrimestre del curso 2019-2020, la asignatura no se ha visto afectada en su periodo de docencia por las medidas excepcionales derivadas de la lucha contra la Covid-19.
} 


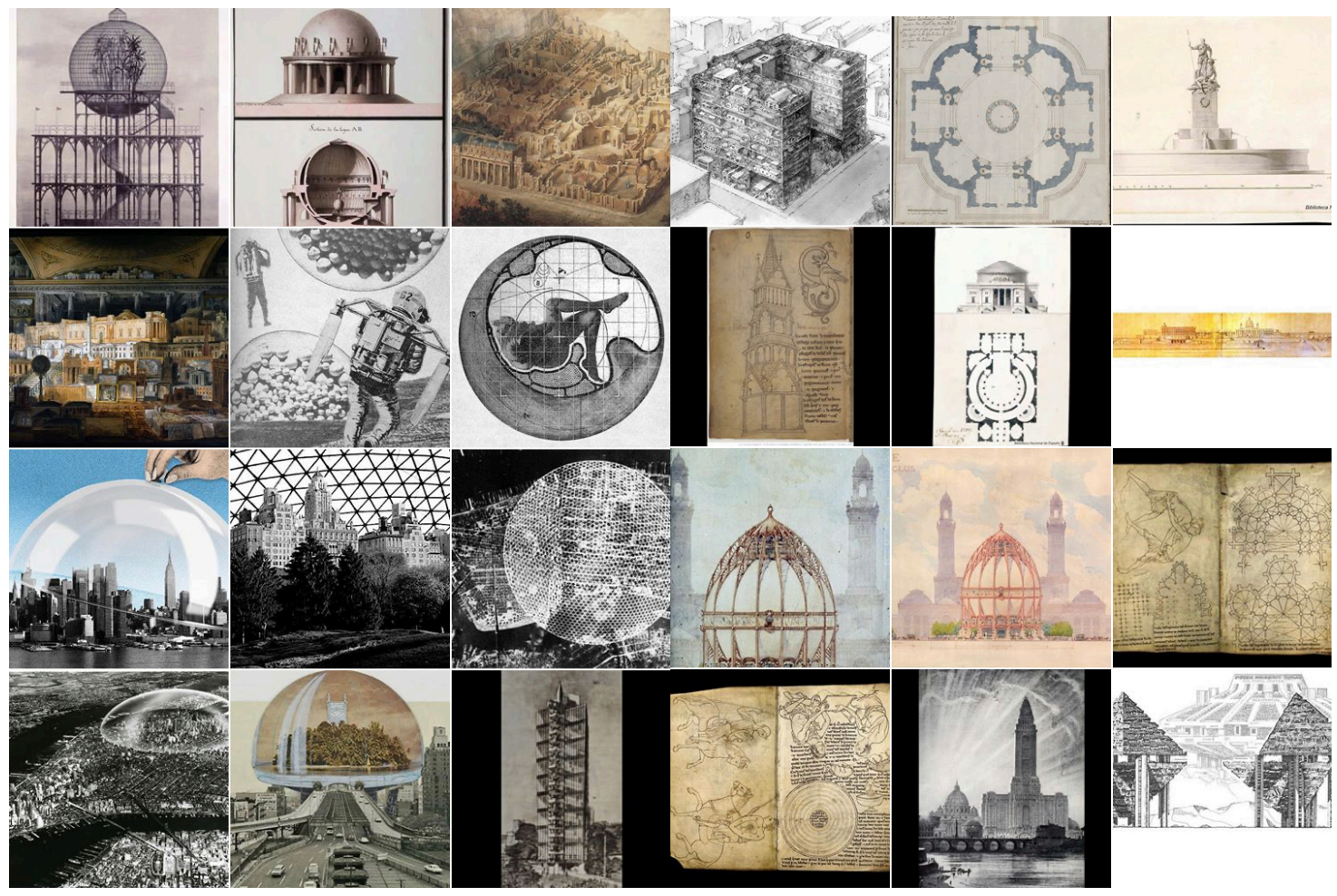

Fig. 7 Algunos ejemplos de arquitecturas dibujadas, de la cuenta del docente 'Arquitecturasenpapel'. Fuente: \#introetsamgrupof (Instagram, 2019)

\section{Conclusiones}

Si bien la propuesta fue inicialmente recibida con entrusiasmo por el grupo, es preciso reconocer que su seguimiento fue desigual a lo largo del curso; en parte tomada como una tarea evaluable más en la evaluación continua de la asignatura. No obstante, del análisis de sus resultados, se deduce que logró alcanzar en parte sus objetivos: el estudiante pudo familiarizarse con la herramienta del 'cuaderno del arquitecto' como archivo de reflexión, análisis y crítica de ejemplos arquitectónicos; además, vinculando esta herramienta al uso de una red social que maneja con soltura, se ofreció una revisión y actualización de la herramienta; el hecho de usar un medio que permite visibilidad también sirvió para dar a conocer a su entorno social (amigos y familia) su nueva dimensión como estudiantes de arquitectura: avances y dedicación; además, la inclusión de las visitas a algunos de los edificios estudiados, fomentó la interacción de los estudiantes entre ellos, generándose pequeños grupos de trabajo y afinidad dentro del grupo general.

El reto de una experiencia como ésta con estudiantes recién ingresados en la Escuela de Arquitectura es que no la entiendan como una carga más en la avalancha de exigencias a la que se ven sometidos. La intención es que asimilen la enseñanza de la herramienta de lo que aquí se ha llamado el 'cuaderno del arquitecto' y que se habitúen a ella, teniéndola como complemento a los medios tradicionales de conocimiento de la arquitectura: libros, revistas, etc. Incorporar para su desarrollo una red social es sólo un medio de actualizar la herramienta propuesta y de acercarla a sus modos de hacer sin que por ello pierda rigor en su objetivo de enseñar a ver arquitectura, en indagar en la idea que siempre tiene tras ella la buena arquitectura.

Esta experiencia apenas ha comenzado, los resultados de este primer contacto sin duda se verán enriquecidos con posteriores ediciones. 


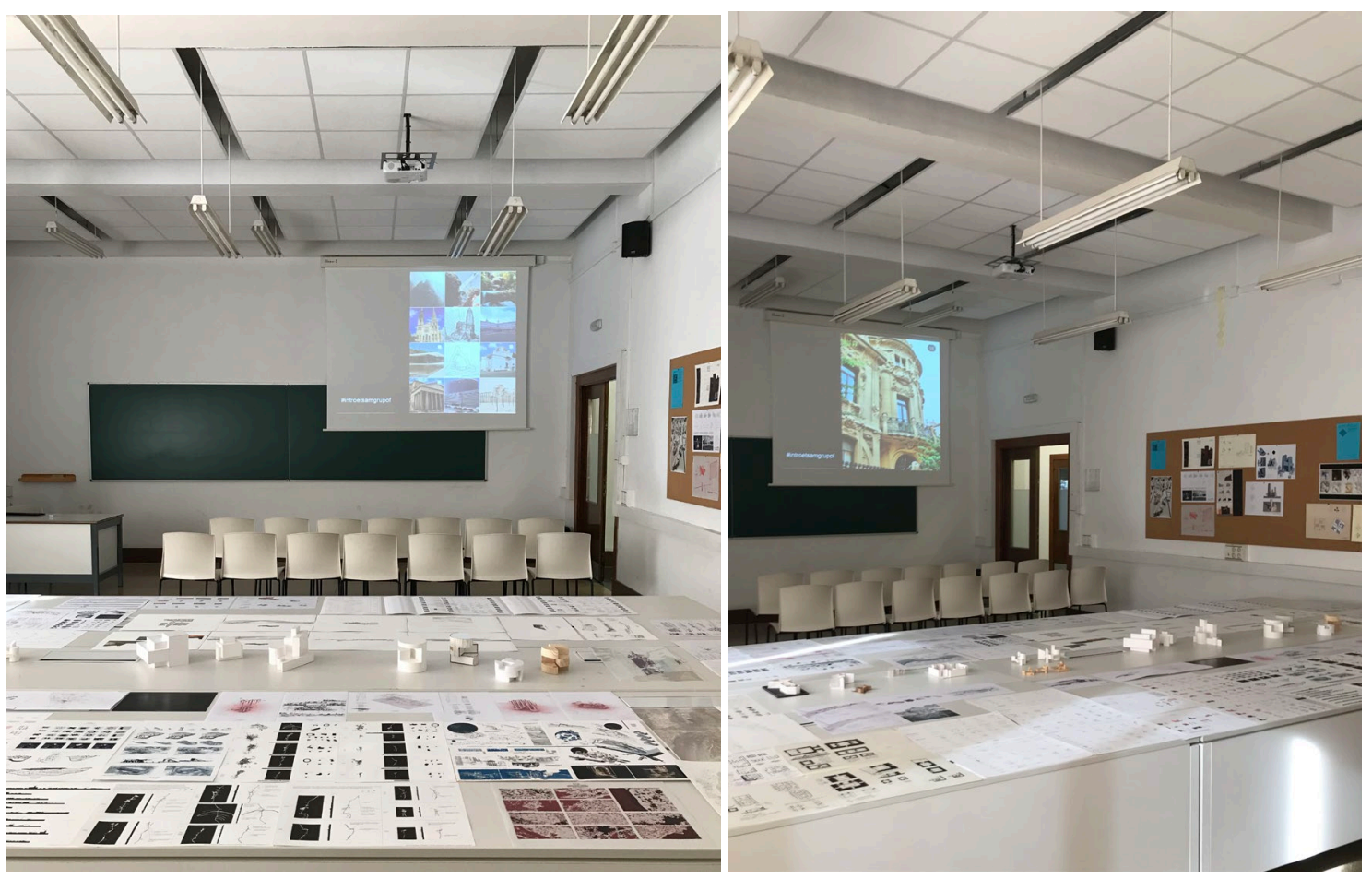

Fig. 8 Presencia del proyecto Arquigramers en las Jornadas de puertas abiertas de la ETSAM, diciembre 2019. Fuente: autor de la comunicación

\section{Bibliografía y referencias web}

ALBA DORADO, M.I. et al. (2019). "Las TIC como apoyo al desarrollo de pensamiento creativo en la docencia de la arquitectura”. En JIDA'19 VII Jornadas sobre Innovación Docente en Arquitectura. Barcelona: Iniciativa Digital Politècnica Oficina de Publicacions Acadèmiques Digitals de la UPC. 544-556.

ALBA DORADO, M.I. (2018). “Innovación docente a través de las Tecnologías de la Información y la Comunicación”. En JIDA'18 VI Jornadas sobre Innovación Docente en Arquitectura. Zaragoza: Iniciativa Digital Politècnica Oficina de Publicacions Acadèmiques Digitals de la UPC y Servicio de publicaciones de la Universidad de Zaragoza. 398-410.

ALEMANY MARTINEZ, C. (2009). "Redes sociales: una nueva via para el aprendizaje". En Cuadernos de Educacion y desarrollo, vol. I, $\mathrm{n}^{\circ}$.1. marzo. <http://www.eumed.net/rev/ced/01/cam4.htm> [Consulta: 29/06/2020].

ALONSO PEREIRA, J.R. et al (2016). "Introducción a la Arquitectura: dando forma al primer contacto". En JIDA'16 IV Jornadas sobre Innovación Docente en Arquitectura. Valencia: Iniciativa Digital Politècnica Oficina de Publicacions Acadèmiques Digitals de la UPC y Editorial Universitat Politècnica de València. 3441.

COMPAGNY, X. (2018). Velázquez: el placer de ver pintura. Lleida, Milenio y Centre d'Art d’Epoca Moderna.

CAMPO BAEZA, A. (1996). La idea construida: la arquitectura a la luz de las palabras. Madrid: Colegio Oficial de Arquitectos.

COLOMINA, B. (2010). Privacidad y Publicidad. La arquitectura moderna como medio de comunicación de masas. Murcia: Cendeac y Colegio Oficial de Arquitectos de Murcia.

DE HARO, J. J. (2010). Redes sociales para la educación. Madrid: Anaya Multimedia.

DÍEZ MEDINA, C. (2018). "Pan, amor y fantasía. Ideas para 'actualizar' la enseñanza de la Composición Arquitectónica”. En JIDA'18 VI Jornadas sobre Innovación Docente en Arquitectura. Zaragoza: Iniciativa 
Digital Politècnica Oficina de Publicacions Acadèmiques Digitals de la UPC y Servicio de publicaciones de la Universidad de Zaragoza. 612-623.

FLORES SOTO, J.A. (2019). "Programa específico de la asignatura Introducción a la Arquitectura", <https://moodle.upm.es/titulaciones/oficiales/course/view.php?id=9226> [consulta: 15/07/2020]

MORENO MORENO, M.P. (2018). "Uso docente de la red social "Instagram" en la asignatura de Proyectos 1". En JIDA'18 VI Jornadas sobre Innovación Docente en Arquitectura. Zaragoza: Iniciativa Digital Politècnica Oficina de Publicacions Acadèmiques Digitals de la UPC y Servicio de publicaciones de la Universidad de Zaragoza. 508-518.

ORDINE, N. (2013). L'utilitá dell'inutile: manifesto. Milano: Bompiani (versión española: La utilidad de lo inútil, Barcelona: Actar, 2013).

ORTEGA y GASSET, J. (1930). Misión de la Universidad, Madrid: Revista de Occidente.

PESQUERA, E. (2019). <https://www.pinterest.es/eduardopesquera/boards/>

RASMUSSEN, S.E. (2004). La experiencia de la arquitectura, Barcelona: Reverté (versión original: Om at opleve arkitektur, Copenhague: G.E.C. Gads Forlag, 1957).

RASMUSSEN, S.E. (2013). Ciudades y edificios descritos con dibujos y palabras, Barcelona: Reverté (versión original: Byer og bygninger: skildret i tegninger ig ord, Copenhague: Fremad, 1949).

RUIZ JARAMILLO, J. et al (2016). "TIC+ABP: dos instrumentos para la innovación docente en Arquitectura". En JIDA'16 IV Jornadas sobre Innovación Docente en Arquitectura. Valencia: Iniciativa Digital Politècnica Oficina de Publicacions Acadèmiques Digitals de la UPC y Editorial Universitat Politècnica de València. 387396.

SENNETT, R. (2008). The Crafsman. New Haven: Yale University Press (versión española: El artesano, Barcelona: Anagrama, 2009).

ZEVI, B. (1951). Saper vedere l'architettura, Milano: Einaudi (versión española: Saber ver la arquitectura, Buenos Aires: Poseidón, 1971). 